\title{
Oxytocin and Collective Bargaining: Propositions for a New Research Protocol
}

\author{
Jean-François Tremblay, Sébastien Rivard, Eric Gosselin \\ Organizational Neuroscience Division (LAPS ${ }^{2}$ ), Industrial Relations Department, Université du Québec en Outaouais, \\ Gatineau, Canada \\ Email: Jean-Francois.Tremblay@uqo.ca,rivs11@uqo.ca, eric.gosselin@uqo.ca
}

How to cite this paper: Tremblay, J.-F., Rivard, S. and Gosselin, E. (2017) Oxytocin and Collective Bargaining: Propositions for a New Research Protocol. American Journal of Industrial and Business Management, 7, 893-909.

https://doi.org/10.4236/ajibm.2017.77063

Received: May 24, 2017

Accepted: June 6, 2017

Published: July 7, 2017

Copyright $\odot 2017$ by authors and Scientific Research Publishing Inc. This work is licensed under the Creative Commons Attribution International License (CC BY 4.0).

http://creativecommons.org/licenses/by/4.0/

\begin{abstract}
This paper contributes to collective bargaining research by providing a causal theoretical biological link path between negotiation behaviors and their substantive and relational results. Specifically, the role of oxytocin is described in light of the scientific knowledge that comes from organizational neurosciences, neuroeconomics and, psychology fields. The properties of the hormone, its place in neuroeconomics research and, their links with the psychology of the collective bargaining processes are discussed to determine guidelines for a new experimental protocol meant to study decision-making processes during collective bargaining. In addition, the conceptual model of strategic negotiations serves as a theoretical framework to consolidate the propositions that can be deduced from the results of the interaction processes in collective bargaining according to two dimensions of the outcome of the negotiations. Finally, the parameters of a new experimental protocol derived from the trust game are presented for the first time. This new game presents a more ecological perspective and is developed to offer a better fit with the specific domain of collective bargaining.
\end{abstract}

\section{Keywords}

Collective Bargaining, Oxytocin, Trust Game, Neuroeconomics, Organizational Neurosciences

\section{Introduction}

Collective bargaining has been the subject of many theoretical contributions from the late 19th century until today [1]. Negotiation theories seek to explain the specific behaviors of negotiators, the dynamics of their exchanges or the results of the negotiations. They originate from disciplines as diverse as economics, sociology, psychology and political sciences [2]. In this regard, industrial re- 
lations researchers have not hesitated to make liberal use of these disciplines in order to enrich the industrial relations field own theories on collective bargaining [3] [4].

The lack of comprehension of the processes underlying the decision-making in the context of collective bargaining has for a long time caused many problems for the researchers trying to understand its dynamics [3]. The process of collective bargaining was a blurry area which contents, often seen as too complex, still resists analysis [5].

Of course, many developments in negotiation theory make it possible to analyze the bargaining process in relation to the environmental variables that influence it and the results generated by the many interactions at the negociationtable. However, these advances struggle to explain and predict accurately many of the behaviors that happen during bargaining, particularly with regard to the dynamics of concessions and trust. Therefore, novel explanatory paradigms are needed to create a new impetus in research on negotiation and to provide a better understanding of the primary factors in play during collective bargaining.

Some of the late discoveries about collective bargaining dynamics come from the use of social psychology theoretical framework. Some interesting findings have been made using this framework about the influence of the psychological characteristics of the negotiator on the bargaining dynamic. Nevertheless, even if we now better understand the bargaining "relation," we feel there is still much more to be understood and it seems that social psychology alone might not be able to reach the bottom of the decision-making process underlying collective bargaining.

To achieve a deeper comprehension of the behavioral schemes and decisions processes of negotiators, we strongly feel that organizational neurosciences can help push research further. Organizational neuroscience (ON) refers to "the application of neuroscientific methods to analyze and understand human behaviors within the applied setting of organizations, which may be at the individual, group, organizational, inter-organizational, and societal levels" [6]. ON is interested in the revealing of the deep roots of behavioral choices at the neuroanatomical, neurofunctional or neuroendocrinal levels. As mentioned by Becker, Cropanzano \& Sanfey. [7], ON is looking for "the most basic building block of behaviors" and what we can call "the neural black box."

Neuroeconomics have already favoured the use of neurosciences theories to better understand the individual's behaviors in different negotiation or "dealing" games. ON could offer an extension of those findings and bring a stronger psychological perspective and insights offering some fundamental explanations on what really happens during collective bargaining.

It appears that the avenue created by neuroscience is promising. In fact, Lewicki \& Polin [8] state:

"Finally, what direction does trust and negotiation research need to go in the near future? One interesting area that could help us to understand these constructs better is the physiological reactions to trust and distrust during a negotiation. Paul Zak, a neuroeconomist, combines knowledge derived 
from neuroscience, economics and psychology to understand what exactly is going on inside the brain when it makes certain decisions. Although not yet specifically applied to negotiation, Zak has explored the effects of the hormone oxytocin, finding it to be associated with trusting. Others research needs to begin addressing what neurochemical dynamics may be related to the many different behaviors exhibited during negotiation."

We are forced to recognize that there are little to no studies which apply ON to the specific topic of collective bargaining. To contribute to the developing knowledge in this new field, we explain, in the context of this article, some commonly accepted properties of oxytocin and expose findings from the literature on the roles this hormone plays both on the level of social interaction and on the substantive results susceptible of characterizing the collective bargaining dynamics. To confirm parallels between more traditional ON results and the effects that are expected from their transposition to the field of industrial relations, a new experimental collective bargaining game specifically dedicated to this purpose is also presented. The purpose of this paper is to propose a new protocol that will allow us to verify the applicability of the current game theory to the neuroscience field through means of hormonal measure applied to specific negotiation context. Since most of the research in this field has focused on hormonal impacts through games that are directed towards general decision-making situations, it appears pertinent to test the validity of the game approach when applied specifically to collective bargaining.

\section{Why Neuroeconomics?}

Since the publication of Adam Smiths' Wealth of Nations, economic theorization has seen a steady increase that peaked in the neoclassical economic revolution [9]. The 1930s saw the rise of the mathematical-logical approaches to patterns and decision-making which paved the way for behavioral economics. The results of this approach brought to light limitations in regard to rational calculations, willpower, and self-interest when combined with elements of psychology [9]. Independently of the field (neoclassical economics or behavioral economics), there have been important variations in responses and limitations on the predictive capability of the theories introduced since. In the 1990s, the discipline of neuroeconomics is ready to emerge between two trends, namely, neuroscientific and neurobehavioral economy which would attempt to correct, with diverging methods, the shortcomings of the previous theories and models [9]. The use of varied strategic games in research allowed tremendous advances in the field of neuroeconomics [10] [11]. Berg, Dickhaut \& McCabe [12], using principles from Nash's work, proposed and tested a game that would be used to verify the existence of reciprocity as a valid decision-making component. That game would be called the "investment game" and would later be known as the trust game. Over the years, many other games and simulations would be created or adapted with roots deep in the realm of behavioral logic that would test the 
realism of the many constraints that have been laid by behavioral economics; which many would blame for their incapacity to account for the human factor at the center of most non-systemic decisions in economics [13]. It is no longer reasonable to pretend that "abstract" human processes such as preferences and beliefs are strictly unobservable, as it has been thought previously [14].

In trying to understand the brain structures and functions responsible for decision-making, the neuroeconomists are attempting to adapt the study of economics to the knowledge gathered on humans and their behaviors. In doing so, they hope to explain many decisions that are considered irrational in regard of a strictly logical interpretation [15]. It seems beneficial, indeed, to give ourselves the tools needed to incorporate, at last, the "human factor" into a science mainly characterized by human decisions in which willpower, empathy, reciprocity, and generosity have been systematically abstracted. Neuroeconomics appears to be just the tool to achieve that reconciliation.

\section{Basic Properties of Oxytocin}

Oxytocin is a nonapeptide hormone that is exclusive to mammalian and is believed to have effects spanning from sexual rhythm regulation to social behaviors structuration. A nonapeptide is a natural biological particle, an oligopeptide composed of amino acids. A peptide is a natural biomolecule that occurs in the body that can have a wide variety of functions depending on their composition [16]. Oxytocin is produced in the supraoptic and paraventricular nuclei of the hypothalamus and is stored, principally, in the pituitary gland [17]. At a primal level, oxytocin regulates birthing functions such as uterus contractions and lactation [18]. Oxytocin is also widely accepted as being of significant importance in many socially related neural processes with regard to social attachment and affiliation [19]. The hormone contributes to basic maternal instincts, namely mother-infant attachment (pair bonding) and social memory (recognition) [20] [21] [22] [23]. Mate selection and monogamous behaviors are also greatly modulated by the actions of oxytocin on the midbrain and amygdala [18] [19] [24]. Additionally, oxytocin is associated with reducing fear, stress and anxiety through inhibition of the aforementioned midbrain and amygdala [21] [25] [26].

Trust appears to be a common denominator when studying the effects of oxytocin on pro-social behaviors. Whether trust is partially achieved as a consequence of fear and stress inhibition or as a specific hormonal response is still open for debate [24] [27]. However, the body of evidence that exists does not permit to cast many doubts on oxytocin as a biological modulator of trust in humans [20] [28] [29].

Oxytocin is either measured directly or indirectly. Direct measurement is achieved through body fluid collection such as blood, urine and, saliva while indirect effect measurement is possible by observing behavioral variations after oxytocin is administered to test subjects [24] [30]. In the body, oxytocin is produced mainly in the brain, but only a portion of the quantity produced enters the bloodstream while the remaining oxytocin is kept within the confines of the 
blood-brain barrier, this calls for careful consideration when deciding on the biological location for direct measurement [24] [31].

On an experimental level, the behavioral effects of oxytocin have been observed on rats, primates and prairie voles mostly, as well as on humans [32] [33]. In non-human mammals, removal of oxytocin production means or inhibition of oxytocin receptors have been shown to induce indifference of the mother towards her progeny and negate sexual interest between mates who had antecedents of regular and frequent sexual contacts [18] [19]. It is obviously impossible to apply to humans many of the experiments that have been performed on animals. Therefore, oxytocin effects have been measured with varying degrees of precision on human subjects through correlations between expected conations and confirmatory testing of the presence of higher oxytocin levels in their system in relation to the oxytocin levels present in control subjects.

\section{Playing Games: The Role of Oxytocin in Trust, Empathy, Pro-Social Behaviors and Risk Aversion}

In 2005, Kosfeld, Heinrichs, Zak, Fischbacher \& Fehr [34] published the results of the first oxytocin study in the young field of neuroeconomics. This was to be a groundbreaking study that would set the basic understandings required to push forward the quest for conceptualizing the role of the human brain in complex decision-making processes [30]. Zak, one of the members of that research team, would become one of the most prominent figures of neuroeconomics, and oxytocin studies. The experiments realized by Zak and his colleagues in 2005 relied on the utilization of the trust game as proposed by Berg, Dickhaut \& McCabe [12] which would be adapted to computerize its stages and the resulting interactions [33] [34].

Following the initial study, there has been an appreciable amount of further research that would build on the foundations laid by Zak and his colleagues. We will review some of the most prominent and defining research on oxytocin in the discipline of neuroeconomics.

Studies based on the trust game-The original study using this particular research protocol is considered a classic in the domain. Berg, Dickhaut \& McCabe [12] would open the door on trust experimentation by devising a game in which the participants would be given the choice to impart any of their money allowance for their participation to an unknown third party. The "investor" would be told that the amount they could choose to give was to be increased threefold on its way to the trustee who would, in turn, be given the chance to give back a discretionary amount to the investor [12]. In staying with the strict principles of Nash's equilibrium, the safest course of action for the investor would be to keep the totality of the initial allowance since there would be no certain way to ensure a return from the trustee. On the other side of the transaction, the trustee, if they were to receive any money, would be expected to then keep the totality of it in their own interest. In such a game design, the conundrum resides in the fact that trust is the only way for both parties to maximize 
their gains. Despite all expectations, very few participants decided to give nothing to the other party, and it has been shown that the trustees who received the higher sum of money would tend to reciprocate more. These conclusions point to the existence of an intrinsic sense of altruism and generosity in participants and that reciprocity can be integrated as part of trust [12].

Kosfeld, Heinrichs, Zak, Fischbacher \& Fehr [34], in their desire to further explain the mechanisms of trust, devised a different version of the trust game by testing for the presence of peripheral oxytocin in the blood of the participants just after decisions that were thought to specifically imply trust. The procedure examined the intentions of the participants in regard to trust as well as their actions to find the presence of a moderate to strong correlation between the levels of peripheral oxytocin in subjects who had the intention to give and in subjects who effectively gave money to the other party [34]. The results, that intranasal administration of oxytocin elevates the level of trust in humans, were congruent to those from Zak, Kurzban \& Matzner [13].

While studying the relation between human trust-based decisions and oxytocin, Kosfeld, Heinrichs, Zak, Fischbacher \& Fehr [34] also introduced another variant of the trust game that was devised to measure the influence of risk aversion behaviors over trust. This version of the game, the risk game, used the same parameters than the trust game with the exception that the sum of money returned to the "investor" was pre-programed by the researchers [35]. By doing so, the investor could not count on any generosity from the part of the "trustee" and had to risk if they decided to transfer money nonetheless [35]. While oxytocin seemed to have a positive effect on trust in the trust game, there were no effects reported on the trust decisions derived from the risk game [34]. These studies show a higher level of oxytocin in individuals who have been on the receiving end of generous offers contrary to when such offers were made randomly by a computer [36]. Also, trustees who gave back higher amount of money to the investor were shown to have peripheral levels of oxytocin superior to their baseline and the mean baseline of all participants and those of the trustees who did not give back [33] [34] [36].

To test this further, Baumgartner, Heinrichs, Vonlanthen, Fischbacher \& Fehr [19], introduced yet another variation in the trust game to account for the participant's reactions to betrayal. In this version, a group of participants is committed to a trust game while a second group is undergoing the risk game. The first group, after the initial transaction (with a human participant), have a 50 50 chances of learning they have been betrayed by their trustee while the second group would have the same odds to be told they had lost money [19]. The striking results of that study is that participants who had received oxytocin would tend to remain trustful even after they were informed of betrayal or of losses. Neuroimaging of the participants showed reduced activity in the amygdala and striatum which are important centers for fear and face-based trust inhibition) [19].

Studies based on the dictator and the ultimatum games-In those games, a 
decision maker (DM1) is entitled with $10 \$$, to be split, in turn, with a second player (DM2) [36]. In the dictator version, DM2 must accept any offer whilst in the ultimatum version, DM2 can reject offers that are below a predetermined amount (the ultimatum) which causes both parties, in the case of rejection by DM2, to lose money [35]. Therefore, in the dictator version, DM1's decisionmaking process is not influenced by the actions of DM2 while, to the contrary, DM2's decisions force DM1 to evaluate their trust position and decision logic before making an offer in the ultimatum version [35]. In the ultimatum game, oxytocin increased generosity by a staggering $80 \%$ while there were no such observable effects for the dictator variant of the experiment [36]. This seems to demonstrate that oxytocin plays a role on empathy and reciprocity. In the ultimatum game, DM1 participants who anticipated the negative response from DM2 and attempted to negate it with more generous offers [36], show that oxytocin could improve pro-social behaviors without affecting subjective awareness [35].

Studies based on the prisoner's dilemma-De Dreu [37] devised a series of experimentations around the prisoner's dilemma to illustrate the possible effects of oxytocin on co-operation. It revealed that, whether isolated prisoners (possibly collaborative participants) were more inclined to cooperate when administered intranasal oxytocin was mainly positively influenced by the participants having physically met prior the experimentation. This would suggest that oxytocin has a strictly limited influence on social behaviors without interfering with emotions or cognitive processes [37]. It appears that social contexts and human interaction must be present for oxytocin to sway the decision of participants in relation to co-operation as shown by further studies using the prisoner's dilemma as a simulation tool [38].

Other prominent research on the subject-Another research in which Zak collaborated showed that physical contact, in this case touching, caressing, hugging and, kissing would produce endogenous oxytocin that would, in turn, facilitate monetary sacrifice. In the same way, customers who are being touched while receiving services are more likely to speak positively of said services or provide higher tips. In that context, survival mechanisms are activated towards friendliness and protection such as in the case of generosity and kindness amongst kin. Women seemed to be even more affected by the effects of physical contacts in a context of monetary sacrifice [39].

A fairly constant element from all the studies mentioned previously is that they all exclusively place the exercise of trust upon money transfers which could bias the response of some participants considering the unique emotional charge associated with money. To counter this possibility, Mikolajczak, Pinon, Lane, de Timary \& Luminet [29], used a protocol in which the subjects would be asked to fill a questionnaire on their sexual habits and fantasies. The questions were specifically designed to invade deeply within the participant's privacy and social sense of shame. Envelopes were provided for the questionnaire that could then be given back and could be sealed and taped to protect from intrusion until the 
answers would be analyzed [29]. The way in which the subjects would give back the envelope and the amount of precaution taken in sealing it would serve as a measure of trust. While $80 \%$ of the control group added tape to seal the envelope, only $7 \%$ did so in the group with that was administered exogenous oxytocin.

Also, an example of research with no monetary basis, Domes, Heinrichs, Michel, Berger \& Herpertz [23], demonstrated that subjects who had received a dose of oxytocin via intranasal injections were more proficient in recognizing emotional states from randomized facial expressions in a "Reading the Mind in The Eyes" test (RMET). Furthermore, the effects of oxytocin would significantly improve with task difficulty, which suggests that the effects of oxytocin are dependent on the context and the difficulty of the task [23] [27]. These results were supported by Van Ijzendoorn and Bakermans-Kranenburg [40] who performed a meta-analysis on all the research mustered on the database Web of Science with the terms "oxytocin," "intranasal" and, "administ" (partial term). The combined effect sizes confirmed that oxytocin administration enhances the recognition of facial expression of emotions and elevates intra-group trust while having no effect on extra-group trust [40].

We touch upon an important point with this last study as there seem to be differences between the effects of oxytocin on intra-group trust and inter-group trust. To further explore that, Ten Velden, Baas, Shalvi, Kret \& De Dreu [40], have performed a double-blind study on participants playing poker. The subjects in the placebo group demonstrated normal competitiveness towards the other players whereas those in the oxytocin groups would settle more readily and compete less with those perceived as members of their play group. In the same vein, there are clues towards confirming the dual nature of oxytocin when implicated in intra vs. inter-group trust processes in another study from De Dreu [37] who states that oxytocin-motivated co-operation is mostly parochial, that is, facilitates in-group favoritism, co-operation towards in-group but not out-group and, defence-motivated non-cooperation towards threatening outsiders [37]. The disparities between research that show intra-group trust and those that do not is a great avenue for future experiments and opens the door to the testing of other hormones simultaneously with oxytocin.

Amongst all the results reviewed on oxytocin-induced trust, some researchers have come forward with surprising and sometimes contradictory results. As we have seen previously, oxytocin is believed to heighten one's ability to accurately identify emotions through facial expressions. However, one study posits that this effect could only be observed in regard to positive emotions and intentions towards others. Israel, Hart \& Winter [42], found that subjects injected with exogenous oxytocin would be less accurate in recognizing threatening intentions via facial expressions than subjects submitted to a placebo. There is even more evidence of the importance of context and situations on the effects of oxytocin in pro-social behaviors as demonstrated by Merolla, Burnett, Pyle, Ahmadi \& Zak [20] who showed that increased oxytocin levels lead to higher interpersonal trust 
and higher political trust in regard to specifics political figures or parties. These results suggested that the subjects' experiences and political conviction can negate the effect of oxytocin when negativity is present and reinforce trust toward a positive bias [20].

Finally, in this brief review of the most prominent research on the effect of oxytocin on trust, we want to turn our gaze towards two more studies that went deeper into understanding the inhibiting factors of trust and oxytocin effects. The first study, realized by Cardoso, Ellenbogen, Serravalle \& Linnen, [26], shows that oxytocin may promote the acquisition of social support in times of distress by increasing self-promoted trust. This supports some of MacDonald \&MacDonald [35] claims that there is a relation between stress and oxytocin and that oxytocin could contribute in reducing or inhibiting the effects of cortisol or its receptors. The same authors, however, go on to point out that the inverse relation could well be true and that cortisol could inhibit the oxytocin receptors [33] [35]. The second research that shall close this section interests itself on the more minute and precise effects of testosterone on oxytocin-induced trust behaviors come from Boksem, Mehta, Van den Bergh, van Son, Trautmann, Roelofs et al. [43] who proposed that testosterone could promote some pro-social behaviors in the absence of stress and competition by inhibiting initial trust but promoting reciprocity. In such a scenario, testosterone would increase defence behaviors but reward caution with heightened mood and generosity when the third party reveals itself to be worthy of trust [43]. This contributes to reaffirming the emerging impression that most hormones are subjected to contexts and situations regarding the specificity of their respective effects [27].

In light of all that precedes, there is definite interest that seems to emerge in the neuroscientific field in regard to resolving a state in which there is a clear lack of consensus concerning the operative mechanisms of decision-making and bargaining-specific behaviors [44]. The existence of folk beliefs on the alleged impacts of some hormones on bargaining behaviors, competitiveness, risk aversion and, aggression is a legacy to the importance the topic has acquired within research and society in general [45]. Therefore, the introduction of new research protocols based on an innovative and more ecological simulation on specific contexts seems to be the most logical and efficacious way to further discoveries.

\section{Proposal for a New Experimental Game: The Negotiator's Game}

Game theory has become an important tool in neurosciences as it involves strategic and dynamic considerations and opposes intelligent opponents likely to be influenced by various circumstances, perceptions and, hormones. To increase the level of realism in decision-making measurements, as well as to promote the congruence between the results obtained from experimentation in the laboratory and the implementation those results within the realm of collective bargaining processes, we believe that the proposal of a new experimental game is desirable, even necessary. In fact, here we offer a contextual background to the game that 
reflects a plausible situation that real negotiators are frequently facing in their practices of labour relations and which aims to be in line with the usual concerns of such practices. It puts into perspective the typical concerns that practitioners may have, namely the achievement of their substantive bargaining objectives and the establishment of the desired social contract, all the while preserving some level of uncertainty about the final outcomes of the negotiations in order to conserve bargaining leverage. In this sense, our game proposal is intended to be a step towards a more detailed understanding of behavioral patterns during negotiation.

Moreover, by putting emphasis on the relational dimension of the negotiations (which is fundamental to collective bargaining), the results of the game may be more influenced by hormonal activity, namely oxytocin, which is deemed important in establishing a confidence bonding and more cooperative social relationships [13].

Our proposal should also constitute a contribution to the field of Behavioral Game Theory as proposed by Camerer \& Chong [46]. To that end, our game also includes some characteristic elements of the trust game while pitting the participants against some typical dynamics of the workplace where the negotiation processes are institutionalized.

Game Description-Each player must decide the best utilization of a punctual sum to the order of $\$ 100,000$ dedicated to labour relations.

Player 1: The employer representative

Player 2: The employee representative

\section{Explanations to the experimenters:}

The players will come face to face after examining separately the parties' position statement and the rules of the game. During the meeting phase of the game, no communication between the players is permitted except for the verbalization of player 1's offer and player 2's response. Both players must first write down their intended strategy before meeting each other. The intended strategy must include, for player 1, the cash value of the proposed amount (between $\$ 0$ and $\$ 100,000$ ) and player 2's anticipated response (accepting or refusing player 1's offer and player 2's decision to renounce to place into play grievances resolutions up to a value of $\$ 300,000$ ). In the case of Player 2, the intended strategy must include the acceptance criterion towards player 1's offer (expressed in \$or by indicating the rejection of the initial offer regardless of the amount proposed or the acceptance of the initial offer regardless of the amount proposed) and the intended decision on the resolution of grievances (total retention, total abandonment or a partial retention ranging from $\$ 1$ and $\$ 299,999$ ) in addition to the player's anticipated proposal (expressed in \$), each player must also indicate whether, in their opinion, the strategy they propose will allow the dispute to be terminated (If they consider that the offer is acceptable to the other party) and what should be the consequences of their strategy on the quality of labor relations (status quo/improvement/deterioration). Players are also advised that, at the time of the verbalization of the offer and the response, they may freely decide 
to make an offer or response that differs from their intended strategy.

Context-The enterprise, Amaza Inc., had an excellent financial year. Therefore, unanticipated cash surplus is available. The management of the company then assigns an amount of $\$ 100,000$ to the employer's representative (player 1) so that they make an appropriate proposal, in terms of labour relations, to the employees' representative (player 2). The quantitative values associated to the proposals made by player 1 and player 2's response will constitute the results of the game. At Amaza Inc., labour relations are judged satisfactory for both sides. The conflicting dynamics between the Employer and the Union do not seem different from what can be observed in other similar organizations, and it varies according to the search for balance between the need to achieve the respective objectives of the parties and maintaining a relative industrial peace.

These days, the main irritant, according to the representatives of both parties, comes from the application of a contractual clause with ambiguous wording in relation to the payment of a bonus. As interpreted by the representative of the employees (player 2), employees who, in the past, were not entitled to the payment of the bonus would now be entitled to receive it. The understanding of the employer's representative (player 1), is that it never was the intention of the parties, during the bargaining process, to give this right to the employees, hence the dispute. Recent discussions concerning a possible agreement to resolve the conflict were held between the employer's representative and the employee's representative. There is a willingness on both sides to settle this dispute without resorting to arbitration. An arbitrator could either render a judgment siding completely with the employer ( $\$ 0$ for the employees) or accessing to the view of the employees ( $\$ 300,000$ for employees) or render any other decision in-between (to an indeterminate amount between $\$ 1$ and \$299,999). Representatives of both parties agree that it is impossible to anticipate the decision ofthe arbitrator.

It should be noted that representatives of the two parties have the same confidence in the merits of their claims. The economic impacts on the organization, compensation of employees and, the climate of labour-management relations are elements that feed the reflection of both parties' representatives with regard to resolving the conflict.

Game process-The employer's representative (player 1) may, to their discretion and in order to encourage the employees' representative (Player 2) to abandon their claims for the application of the disputed clause, offer employees a lump sum between $\$ 1$ and $\$ 100,000$ or decide to keep the amount available to devote to other purposes in the future. Where applicable, the employee's representative decides whether to accept the amount offered. Likewise, and independently of their previous decision, they can decide to drop or not, in whole or in part, their monetary claims regarding the application of the disputed contractual clause.

Different choice combinations are possible. These combinations are, at the extremes: 1) the employer's representative formulates no offer and the employee representative abandons his claims for the disputed clause (most favorable sce- 
nario for the employer); 2) the employer's representative offers $\$ 100,000$ to the representative of the employees that they accept without reducing their monetary claims regarding the application of the bonus clause (the worst-case scenario to the employer). Any combination of offers and counter offers are possible between those extremes.

\section{Theoretical Framework}

In many ways, the effects associated with oxytocin in study-based games tend to fall logically within the well-known phenomena of the dynamics of negotiation. One can think of the reciprocal effect in the dynamics of the concession, the hazards of establishing a relationship of trust between the protagonists and, a continuum of attitudes ranging from confrontation to co-operation [2]. Beyond environmental contingencies, bargaining power and cognitive choices of participants in the negotiations, it seems sensible to posit in the light of the literature presented in this article that the actions at the negotiation table are influenced by oxytocin. Therefore, one can postulate that, like cognitive mechanisms, the hormone plays an active role in determining the choices of the negotiator.

In order to test this postulate and deduct its expected effects in terms of negotiation dynamics, we retain the model of strategic negotiations [47]. The model has three dimensions: 1 ) the forces influencing the choice of negotiators, that is, the desirability of change (objectives regarding the rules and, under-agreement social contract) and feasibility of change (the expected or planned responses, the relative bargaining power of each party and, the other factors influencing the negotiators) are the inputs of the "system" of strategic negotiation. 2) Conversion of input mechanisms previously identified, namely the interaction between trading strategies (escape, forcing and fostering), the negotiation process (distributive or integrative negotiation, shaping inter-group attitudes and managing internal differences) and, finally, the negotiation structures. Thelast element of the model, 3) refers to the output of the negotiation process, specifically the rules under the agreement (contract terms, normative and, monetary) and the facets of the social contract that reflect the nature of the relationship that the parties maintain with each other.

Walton, Cutcher-Gershenfeld \& McKersie [47], also explain the strategic standard alignments for each of the two main trading strategies, namely those of forcing and fostering. The authors state that forced negotiation, which refers to a "win-lose" dynamic relies on a distributive negotiation process. The necessary presence of an internal consensus while exploiting the intra-organizational disputes of the opposing party constitutes the main challenge in terms of managing internal differences. Similarly, it becomes the task of the negotiators who use this strategy to seek, in the context of shaping the process of inter-group attitudes, to destabilize and create cognitive dissonance in the other party.

Inversely, negotiators who wish to adopt a fostering negotiation strategy ("win-win" approach) will draw on assumptions associated with integrative negotiation and seek fluidity and accuracy in the information exchange. In addi- 
tion, the search for homogeneity in the respective positions of each party (intra-organizational consensus amongst all parties) will be at the heart of the negotiators' concerns. Finally, negotiators will attempt to create or consolidate a climate of mutual trust in order to achieve and maintain cooperative negotiations.

Using the game proposed in this article, we are able to neutralize the environmental variables, bargaining power and the components specific to bargaining structures to focus mainly on the strategies of the protagonists and their outputs with regard to substantive results and the social contract state. It will be left to appreciate if a larger concern for the social contract (and, therefore, prosocial behavior), greater confidence in each other and, seeking greater reciprocity in the offers and earnings are dependent on the influence of the hormone or stimulates the same hormone. Moreover, it appears that the negotiating dynamics proposed by the model are consistent with the findings from the literature on oxytocin. The link between the strategic behaviors of the negotiators and the role of the hormone is thus reinforced.

The game we propose differs from the trust game in several points. Firstly, the winning/losing conditions do not rest on actual currencies that the participants will get to keep after the experimentation. Since collective bargaining can often be done by "proxies," namely negotiators that have no direct stakes in the results, it is important to verify that a game resting on such issues can still be influenced by oxytocin levels even if there is no actual money involved for the participants. Secondly, the proposed game will introduce the participants and allow them to partake face to face, with very limited interactions, which could illustrate new dynamics. For example, will there be significant increases of oxytocin that could have an impact on trust when participants of opposite sex will be in the presence of one another? Lastly, would an increase in trust be enough to effect measurable changes in both substantive and relational axis of the strategic negotiation?

We posit that the first step in obtaining new knowledge about the collective bargaining process through the discipline of organizational neurosciences is to first validate a game protocol that does not stray too far from the classic simulations. In this, the game we propose introduces a minimal number of modifications from the trust game, and should allow for a proper exploration of the effects of oxytocin on the collective bargaining cognitive processes.

\section{Propositions}

Keeping in line with the theoretical predictions of the Walton, Cutcher-Gershenfeld \& McKersie [47] model and the experimental findings of Zak, Kurzban \& Matzner [13] we formulate the following propositions:

P1-The lower player 1's level of oxytocin, the more that player is susceptible to give low importance to the social contract, not display trust, adopt a forcing strategy and aim to minimize the amount offered to player 2.

P2-The higher player 1's level of oxytocin, the higher that player is suscepti- 
ble to give importance to the social contract, display trust, adopt a fostering strategy and maximize the amount offered to player 2.

P3-The lower player 1's level of oxytocin, the more that player is susceptible to choose a forcing strategy and expect player 2 to:

-Refuse an offer inferior to $\$ 100000$

-Refuse to concede on their original claims

P4-The higher player 1's level of oxytocin, the more that player is susceptible to choose a fostering strategy and expect player 2 to:

-Accept an offer inferior to $\$ 100,000$

-Accept to concede on their original claims

P5-The more player 1's offer is perceived as generous, the higher player 2's level of oxytocin is elevated and the more they will tend to reciprocate on player l's offer by renouncing on their initial claims.

P6-The less player 1's offer is perceived as generous, the lower player 2's level of oxytocin, the more they will tend to oppose player 1's offer by holding on to their initial claims.

\section{Conclusions}

In reviewing the previous observations and results of the studies presented, we can extract a number of postulates that could be useful in circumventing difficulties that we invoqued on the subject of this work and orient further research.

Firstly, the link between oxytocin and trust seems to be solid. There are very few publications that contradict this. When there is a debate, it resides mainly amongst the specific parameters of its operationalization. Secondly, there is no "chicken or egg" conundrum in the link between oxytocin and trust since the endogenous effects have been validated with exogenous administrations of oxytocin and found to produce the same effects. This allows us to express confidence, through consistency, in the actual body of knowledge on the subject. Thirdly, all variations between the different results, when it comes to experimentations based on games or double-blind experimentation, seem to be explained by the contextual nature of the effects of the hormone. It appears to us that some of the debates could be quenched with an experimental protocol that incorporates more ecological parameters into the test while still being controlled and backed by a solid theoretical foundation. Lastly, there is evidence that other hormones could play a role in the relation between trust and oxytocin. It might be required to include further measurements to control the effects of testosterone and cortisol simultaneously in the future.

These conclusions suggest that research on oxytocin could be an ideal avenue to further our understanding of decision-making processes. The trust game has been a valid and much useful tool in trying to understand the impacts of trust on financial and strategic decisions. The adaptation of this game to a collective bargaining context should allow us to disprove or confirm the applicability of what is already known from the field of behavioral economics to the neuroendocrinological mechanisms underlying negotiations. The natural ecological limits im- 
posed by the rules of labour relations seem to be an ideal background on which to shape the creation of a new experimental protocol. Along the experimentation, it should be proven easy to introduce different variations to the protocol while still being strictly guided by the theoretical models of strategic negotiations. This new protocol also includes a new dimension that should impose an implicit constraint that is nonexistent in the classic trust game: preoccupations for the social contract between the parties which, in turns, brings the potentiality for relations-based concerns within the organization.

There is much still left to be done in the quest for a complete understanding of the individual nature of the decisions that intervene in the collective bargaining act. It appears undeniable that there is a biological component in every human action which can impact, to varying degrees, on cognitive processes. The current state of the accumulated knowledge on the subject suggests that we may have hit a wall preventing us from progressing much further using only the classical methods. Organizational neurosciences seem to be a door in that wall as it opens a widely unexplored field of seldom known sources of influence and explanations to the human minds and its operationalization. The field allows for greater objectivity into the decision-making process of negotiators not limited by the incapacity to go beyond the simple observation of the process.

\section{References}

[1] Tremblay, J.F. and Bergeron, J.G. Perspectives Théoriques sur la Préparation de la Négociation Collective. Transformations du Monde du Travail: Regards Interdisciplinaires en Relations Industrielles. Presses de l'Université du Québec, 227-250.

[2] Lewicki, Saunders, D.M. and Barry, B. (2014) Negotiation. McGraw-Hill Education, New York.

[3] Kochan, T.A. (1993) Teaching and Building Middle Range Industrial Relations Theory. Industrial Relations Theory: Its Nature, Scope, and Pedagogy, 4, 353.

[4] Kaufman, B.E. (1993) The Origins \& Evolution of the Field of Industrial Relations in the United States. ILR Press.

[5] Bacharach, S.B. and Lawler, E.J. (1981) Bargaining: Power, Tactics and Outcomes.

[6] Butler, M.J. (2014) Operationalizing Interdisciplinary Research-A Model of CoProduction in Organizational Cognitive Neuroscience. Frontiers in Human Neuroscience, 7. https://doi.org/10.3389/fnhum.2013.00720

[7] Becker, W.J., Cropanzano, R. and Sanfey, A.G. (2011) Organizational Neuroscience: Taking Organizational Theory inside the Neural Black Box. Journal of Management. https://doi.org/10.1177/0149206311398955

[8] Lewicki and Polin (2013) Trust and Negotiation (Handbook of Research on Negotiation). Edward Elgar Publishing, Northampton.

[9] Glimcher, P.W. and Fehr, E. (2013) Neuroeconomics: Decision Making and the Brain. Academic Press.

[10] Johnson, N.D. and Mislin, A.A. (2011) Trust Games: A Meta-Analysis. Journal of Economic Psychology, 32, 865-889. https://doi.org/10.1016/j.joep.2011.05.007

[11] Sanfey, A.G. (2007) Social Decision-Making: Insights from Game Theory and Neuroscience. Science, 318, 598-602. https://doi.org/10.1126/science.1142996

[12] Berg, J., Dickhaut, J. and McCabe, K. (1995) Trust, Reciprocity, and Social History. 
Games and Economic Behaviour, 10, 122-142.

https://doi.org/10.1006/game.1995.1027

[13] Zak, Kurzban, R. and Matzner, W.T. (2005) Oxytocin Is Associated with Human Trustworthiness. Hormones and Behavior, 48, 522-527.

[14] Fehr, E. and Camerer, C.F. (2007) Social Neuroeconomics: The Neural Circuitry of Social Preferences. Trends in Cognitive Sciences, 11, 419-427.

[15] Bossaerts, P. and Murawski, C. (2015) From Behavioural Economics to Neuroeconomics to Decision Neuroscience: The Ascent of Biologyin Research on Human Decision-Making. Current Opinion in Behavioural Sciences, 5, 37-42.

[16] Ochsner, K. and Kosslyn, S.M. (2013) The Oxford Handbook of Cognitive Neuroscience, Volume 1: Core Topics: OUP USA.

[17] Ross, H.E., Cole, C.D., Smith, Y., Neumann, I.D., Landgraf, R., Murphy, A.Z., et al. (2009) Characterization of the Oxytocin System Regulating Affiliative Behavior in Female Prairie Voles. Neuroscience, 162, 892-903.

[18] Insel, T.R. and Fernald, R.D. (2004) How the Brain Processes Social Information: Searching for the Social Brain. Annual Review of Neuroscience, 27, 697-722.

https://doi.org/10.1146/annurev.neuro.27.070203.144148

[19] Baumgartner, T., Heinrichs, M., Vonlanthen, A., Fischbacher, U. and Fehr, E. (2008) Oxytocin Shapes the Neural Circuitry of Trust and Trust Adaptation in $\mathrm{Hu}$ mans. Neuron, 58, 639-650.

[20] Merolla, J., Burnett, G., Pyle, K., Ahmadi, S. and Zak, P. (2013) Oxytocin and the Biological Basis for Interpersonal and Political Trust. Political Behavior, 35, 753776. https://doi.org/10.1007/s11109-012-9219-8

[21] Striepens, N., Kendrick, K.M., Maier, W. and Hurlemann, R. (2011) Prosocial Effects of Oxytocin and Clinical Evidence for Its Therapeutic Potential. Frontiers in Neuroendocrinology, 32, 426-450.

[22] Campbell, A. (2010) Oxytocin and Human Social Behavior. Personality and Social Psychology Review, 14, 281-295. https://doi.org/10.1177/1088868310363594

[23] Domes, G., Heinrichs, M., Michel, A., Berger, C. and Herpertz, S.C. (2007) Oxytocin Improves "Mind-Reading" in Humans. Biological Psychiatry, 61, 731-733.

[24] Sue Carter, C., Pournajafi-Nazarloo, H., Kramer, K.M., Ziegler, T.E., White-Traut, R., Bello, D., et al. (2007) Oxytocin. Annals of the New York Academy of Sciences, 1098, 312-322. https://doi.org/10.1196/annals.1384.006

[25] Yao, S., Zhao, W., Cheng, R., Geng, Y., Luo, L. and Kendrick, K.M. (2014) Oxytocin Makes Females, But Not Males, Less Forgiving Following Betrayal of Trust. The International Journal of Neuropsychopharmacology, 17, 1785-1792. https://doi.org/10.1017/S146114571400090X

[26] Cardoso, C., Ellenbogen, M.A., Serravalle, L. and Linnen, A.-M. (2013) Stress-Induced Negative Mood Moderates the Relation Between Oxytocin Administration and Trust: Evidence for the Tend-and-Befriend Response to Stress? Psychoneuroendocrinology, 38, 2800-2804.

[27] Bartz, J.A., Zaki, J., Bolger, N. andOchsner, K.N. (2011) Social Effects of Oxytocin in Humans: Context and Person Matter. Trends in Cognitive Sciences, 15, 301-309.

[28] Declerck, C.H., Boone, C. and Emonds, G. (2013) When Do People Cooperate? The Neuroeconomics of Prosocial Decision-Making. Brain and Cognition, 81, 95-117.

[29] Mikolajczak, M., Pinon, N., Lane, A., de Timary, P. and Luminet, O. (2010) Oxytocin Not Only Increases Trust When Money Is at Stake, But Also When Confidential Information Is in the Balance. Biological Psychology, 85, 182-184.

[30] MacDonald, Dadds, M.R., Brennan, J.L., Williams, K., Levy, F. and Cauchi, A.J. 
(2011) A Review of Safety, Side-Effects and Subjective Reactions to Intranasal Oxytocin in Human Research. Psychoneuroendocrinology, 36, 1114-1126.

[31] Horvat-Gordon, M., Granger, D.A., Schwartz, E.B., Nelson, V.J. and Kivlighan, K.T. (2005) Oxytocin Is Not a Valid Biomarker When Measured in Saliva by Immunoassay. Physiology \& Behavior, 84, 445-448.

[32] Purves, D. (2013) Principles of Cognitive Neuroscience. Macmillan Education.

[33] Zak (2012) The Moral Molecule: How Trust Works. Penguin Publishing Group.

[34] Kosfeld, M., Heinrichs, M., Zak, P.J., Fischbacher, U. and Fehr, E. (2005) Oxytocin Increases Trust in Humans. Nature, 435, 673-676. https://doi.org/10.1038/nature03701

[35] MacDonald and MacDonald, T.M. (2010) The Peptide That Binds: A Systematic Review of Oxycotin and Its Pro-Social Effects in Humans. Harvard Review of Psychiatry, 18, 1-21. https://doi.org/10.3109/10673220903523615

[36] Zak, P.J., Stanton, A.A. and Ahmadi, S. (2007) Oxytocin Increases Generosity in Humans. PLoS ONE, 2, e1128. https://doi.org/10.1371/journal.pone.0001128

[37] De Dreu, C.K.W. (2012) Oxytocin Modulates Co-Operation within and Competition between Groups: An Integrative Review and Research Agenda. Hormones and Behavior, 61, 419-428.

[38] Declerck, C.H., Boone, C. and Kiyonari, T. (2010) Oxytocin and Co-Operation under Conditions of Uncertainty: The Modulating Role of Incentives and Social Information. Hormones and Behavior, 57, 368-374.

[39] Morhenn, V.B., Park, J.W., Piper, E. and Zak, P.J. (2008) Monetary Sacrifice among Strangers Is Mediated by Endogenous Oxytocin Release after Physical Contact. Evolution and Human Behavior, 29, 375-383.

[40] Van Ijzendoorn, M.H. and Bakermans-Kranenburg, M.J. (2012) A Sniff of Trust: Meta-Analysis of The Effects of Intranasal Oxytocin Administration on Face Recognition, Trust to In-Group, and Trust to Out-Group. Psychoneuroendocrinology, 37, 438-443.

[41] Ten Velden, F.S., Baas, M., Shalvi, S., Kret, M.E. and De Dreu, C.K.W. (2014) Oxytocin Differentially Modulates Compromise and Competitive Approach But Not Withdrawal to Antagonists from Own vs. Rivaling Other Groups. Brain Research, 1580, 172-179.

[42] Israel, S., Hart, E. and Winter, E. (2014) Oxytocin Decreases Accuracy in the Perception of Social Deception. Psychological Science, 25, 293-295. https://doi.org/10.1177/0956797613500794

[43] Boksem, M.A.S., Mehta, P.H., Van den Bergh, B., van Son, V., Trautmann, S.T., Roelofs, K., et al. (2013) Testosterone Inhibits Trust but Promotes Reciprocity. Psychological Science, 24, 2306-2314. https://doi.org/10.1177/0956797613495063

[44] Mehta, P.H., Mor, S., Yap, A.J. and Prasad, S. (2015) Dual-Hormone Changes Are Related to Bargaining Performance. Psychological Science, 26, 866-876. https://doi.org/10.1177/0956797615572905

[45] Eisenegger, C., Naef, M., Snozzi, R., Heinrichs, M. and Fehr, E. (2010) Prejudice and Truth about the Effect of Testosterone on Human Bargaining Behaviour. Nature, 463, 356-359. https://doi.org/10.1038/nature08711

[46] Camerer, C.F., Ho, T.-H. and Chong, J.-K. (2002) Sophisticated ExperienceWeighted Attraction Learning and Strategic Teaching in Repeated Games. Journal of Economic Theory, 104, 137-188. https://doi.org/10.1006/jeth.2002.2927

[47] Walton, R.E., Cutcher-Gershenfeld, J. and McKersie, R.B. (2000) Strategic Negotiations: A Theory of Change in Labor-Management Relations. ILR Press. 
Submit or recommend next manuscript to SCIRP and we will provide best service for you:

Accepting pre-submission inquiries through Email, Facebook, LinkedIn, Twitter, etc. A wide selection of journals (inclusive of 9 subjects, more than 200 journals)

Providing 24-hour high-quality service

User-friendly online submission system

Fair and swift peer-review system

Efficient typesetting and proofreading procedure

Display of the result of downloads and visits, as well as the number of cited articles Maximum dissemination of your research work

Submit your manuscript at: http://papersubmission.scirp.org/

Or contact ajibm@scirp.org 